\title{
Comparison of the Efficacy of Polaprezinc Plus Proton Pump Inhibitor and Rebamipide Plus Proton Pump Inhibitor Treatments for Endoscopic Submucosal Dissection-induced Ulcers
}

\author{
Da Hyun Jung, MD, * Jun Chul Park, MD, PhD, * Yong Chan Lee, MD, PhD, * \\ Sang Kil Lee, MD, PhD, * Sung Kwan Shin, MD, PhD,* \\ Hyunsoo Chung, MD, † Jae Jun Park, MD, PhD, ${ }^{*}$ Jie-Hyun Kim, MD, PhD, $\neq$ \\ Young Hoon Youn, MD, PhD, $\ddagger$ and Hyojin Park, MD, PhD $\ddagger$
}

\begin{abstract}
Goals: We assessed the efficacy of polaprezinc plus proton pump inhibitor (PPI) treatment for endoscopic submucosal dissection (ESD)induced ulcer healing compared with rebamipide plus PPI treatment.

Background: ESD has been widely used as a local treatment option that cures gastric neoplasms. However, it causes large and deep artificial ulcers, and there are no guidelines with regard to the optimal treatment durations and drug regimens for ESD-induced ulcers. Polaprezinc is effective for promoting ulcer healing and helps enhance the quality of ulcer healing.
\end{abstract}

Study: Two hundred ten patients with ESD-induced ulcers were randomly allocated to treatment with polaprezinc $(150 \mathrm{mg} / \mathrm{d})$ plus pantoprazole $(40 \mathrm{mg} / \mathrm{d})$ or treatment with rebamipide $(300 \mathrm{mg} / \mathrm{d})$ plus pantoprazole $(40 \mathrm{mg} / \mathrm{d})$. We evaluated the ulcer healing rate and condition of the ulcer at 4 weeks after dissection. The $\chi^{2}$ or Fisher exact test and the Student $t$ test were used.

Results: The ulcer healing rates at 4 weeks after dissection in the polaprezinc plus pantoprazole treatment group were not inferior compared with those in the rebamipide plus pantoprazole treatment group, both in the intention-to-treat analysis $(90.3 \%$ and $91.4 \%$, respectively, $P=0.523)$ and per-protocol analysis $(89.9 \%$ and $91.1 \%$, respectively, $P=0.531$ ). The short procedure time was an independent predictive factor for a high ulcer healing rate (odds ratio: 0.975 ; $95 \%$ confidence interval: $0.958-0.993 ; P=0.006$ ).

Received for publication July 26, 2019; accepted March 17, 2020.

From the *Department of Internal Medicine, Yonsei University College of Medicine; $†$ Department of Internal Medicine and Liver Research Institute, Seoul National University College of Medicine; and tDepartment of Internal Medicine, Gangnam Severance Hospital, Yonsei University College of Medicine, Seoul, Korea.

Funded by SK Chemicals.

The authors declare that they have nothing to disclose.

Address correspondence to: Yong Chan Lee, MD, PhD, Department of Internal Medicine, Yonsei University College of Medicine, 50-1 Yonsei-ro, Seodaemun-gu, Seoul 03722, Korea

(e-mail: leeyc@yuhs.ac); Hyojin Park, MD, PhD, Department of Internal Medicine, Gangnam Severance Hospital, Yonsei University College of Medicine, 211 Eonjuro, Gangnam-gu, Seoul 06283, Korea (e-mail: hjpark21@yuhs.ac).

Supplemental Digital Content is available for this article. Direct URL citations appear in the printed text and are provided in the HTML and PDF versions of this article on the journal's website, www.jcge. com.

Copyright (C) 2021 The Author(s). Published by Wolters Kluwer Health, Inc. This is an open-access article distributed under the terms of the Creative Commons Attribution-Non Commercial-No Derivatives License 4.0 (CCBY-NC-ND), where it is permissible to download and share the work provided it is properly cited. The work cannot be changed in any way or used commercially without permission from the journal.

DOI: $10.1097 / \mathrm{MCG} .0000000000001357$
Conclusion: The polaprezinc plus PPI treatment showed noninferiority to rebamipide plus PPI treatment in the ulcer healing rate at 4 weeks after ESD.

Key Words: polaprezinc, endoscopic submucosal dissection, ulcer

( Clin Gastroenterol 2021;55:233-238)

E ndoscopic submucosal dissection (ESD) is widely used to - treat gastric neoplasms. ${ }^{1}$ Although ESD shows higher enbloc and curative resection rates than endoscopic mucosal resection, it causes larger and deeper artificial ulcers than endoscopic mucosal resection. A larger artificial ulcer is a risk factor of delayed bleeding. ${ }^{2}$

Therefore, the management of artificial ulcers is important. Proton pump inhibitors (PPIs), histamine-2 receptor antagonists, antacids, and mucosal protective drugs may heal artificial ulcers induced by ESD faster. However, there is no consensus with regard to optimal drugs and the proper duration. PPIs have been known to be the most effective type of drug for the treatment of artificial ulcers. However, in some studies, PPI monotherapy was not sufficient to heal ESD-induced artificial ulcers. ${ }^{3}$

Rebamipide is a mucoprotective agent that accelerates ulcer healing. ${ }^{4}$ Rebamipide increases the glycoprotein content in gastric mucus and decreases reactive oxygen species. ${ }^{5,6}$ Therefore, it protects the gastric mucosa and promotes quality of ulcer healing. Polaprezinc, a zinc-L-carnosine chelate compound, is used for the treatment of gastric ulcer. It exerts gastric mucosal protection and promotes ulcer healing through antioxidant activity. ${ }^{7}$ There is a report that polaprezinc plus PPI treatment prevented protrusion of the ulcer base during the healing of ESD-induced artificial ulcers compared with PPI monotherapy. ${ }^{8}$ In this study, we aimed to compare the rate of ulcer healing between polaprezinc and PPI combination therapy and rebamipide and PPI combination therapy.

\section{METHODS}

\section{Patients}

Between November 2014 and April 2016, we enrolled patients who underwent ESD for gastric neoplasms for this prospective, randomized, controlled study, which was conducted at the 2 hospitals of Yonsei University College of Medicine. Written informed consent was obtained from all patients before study enrollment. Inclusion criteria were (1) age 20 to 80 years and (2) patients pathologically diagnosed 
with gastric adenoma or cancer who were eligible for ESD. Exclusion criteria were (1) use of medication such as PPIs, histamine-2 receptor antagonists, or other mucosal protective drugs within 2 weeks before enrollment; (2) those who expected to take nonsteroidal anti-inflammatory drugs (including aspirin) and steroids within 2 weeks before enrollment; (3) history of upper gastrointestinal surgery; (4) history of allergy to a PPI, rebamipide, or polaprezinc; and (5) significant cardiovascular, renal, hepatic, neurotic, or psychological disorders.

The institutional review board of Severance and Gangnam Severance Hospitals approved this study. This study was registered with clinicaltrials.gov (identifier 02243618).

\section{Study Design (Sample Size, Randomization, and Follow-up)}

On the basis of a previous study, ${ }^{9}$ the ulcer healing rate of the PPI and rebamipide arm after 4 weeks of administration was $95 \%$, and the SD was $11 \%$. The sample size was calculated by using an $\alpha$ error of 0.025 , statistical power of 0.9 , and 1-sided test. The calculated sample size was 103 lesions in each arm, for a total of 218 patients, allowing for a $5 \%$ dropout rate determined by assuming a noninferiority margin of $5 \%$ and standard deviation of $11 \%$.

A single study coordinator performed the randomization process using a computer-generated randomization table. Patients were randomly assigned to one of 2 groups (PPI plus rebamipide or PPI plus polaprezinc). All patients received an intravenous injection of $40 \mathrm{mg}$ of pantoprazole twice on the first 2 days after ESD. Beginning on the third day after ESD, the patients in the PPI plus rebamipide group took $40 \mathrm{mg}$ of oral pantoprazole once a day and $100 \mathrm{mg}$ rebamipide 3 times a day for 28 days; the patients in the PPI plus polaprezinc group took $40 \mathrm{mg}$ of oral pantoprazole once a day and $75 \mathrm{mg}$ of polaprezinc twice a day for 28 days.
The enrolled patients underwent follow-up endoscopy at 4 weeks after ESD to evaluate the degree of ulcer healing. The adverse events and compliance with taking the drugs were evaluated at 4 weeks. The patients who had low medication compliance $(<80 \%)$ and took the prohibited drugs mentioned in the exclusion criteria were excluded in the per-protocol analysis.

\section{Evaluation of the Primary and Secondary Outcomes}

The primary endpoint was the ulcer healing rate at 4 weeks, which was calculated as follows: [(initial ulcer area-ulcer area at $4 \mathrm{wk}) \times 100$ /initial ulcer area]. The diameter of the ulcer was measured by using an endoscopic forcep (Olympus Optical Co. Ltd, Tokyo, Japan) (Supplementary Fig. 1, Supplemental Digital Content 1, http://links.lww.com/JCG/A558). Ulcer areas were calculated by multiplying these 2 diameters. The secondary endpoints were the proportion of patients based on the ulcer stage, which was scarring, and quality of ulcer healing at 4 weeks. The ulcer stage was assessed by using the classification of Sakita and Fukutomi, ${ }^{10}$ and described as active (A1, A2), healing $(\mathrm{H} 1, \mathrm{H} 2)$, and scarring $(\mathrm{S} 1, \mathrm{~S} 2)$ stages.

\section{Statistical Analysis}

The $\chi^{2}$ test or Fisher exact test was used to compare categorical parameters. The Student $t$ test was used to compare continuous variables. Risk factors affecting the ulcer healing rate were evaluated using logistic regression analysis. The accepted significance level was $P$-value $<0.05$. All statistical analyses were performed using SPSS version 23.0 for Windows (SPSS Inc., Chicago, IL).

\section{RESULTS}

\section{Baseline Characteristics}

Among 218 patients, 109 patients each were randomly allocated to the PPI plus rebamipide and PPI plus polaprezinc



FIGURE 1. Flow chart of patients. PP indicates per-protocol. 
group. Among them, 8 patients dropped out of the study because of delayed bleeding ( 1 patient in the PPI plus rebamipide group), additive surgery owing to a positive resection margin, or withdraw of informed consent. Thus, the 210 patients (106 in the PPI plus rebamipide group and 104 in the PPI plus polaprezinc group) who completed the study protocol were included in the full analysis. After excluding patients on the basis of study protocols such as $<80 \%$ drug compliance or taking forbidden drugs, the data for 100 patients in the PPI plus rebamipide group and 99 patients in the PPI plus polaprezinc group were used in the per-protocol analysis (Fig. 1). There were no significant differences between the 2 groups in terms of age and sex, and histopathology, location, gross appearance, and diameter of the tumor and in terms of ulcer size, en-bloc resection rate, and procedure time. Helicobacter pylori infection developed significantly more in the PPI plus rebamipide group than in the PPI plus polaprezinc group (Table 1).

\section{Healing Rates of latrogenic Ulcers at 4 Weeks After ESD}

The average ulcer healing rate of all patients at 4 weeks after ESD was $90.5 \%$. The ulcer healing rate was not significantly

TABLE 1. Baseline Characteristics of the Enrolled Patients

\begin{tabular}{|c|c|c|c|}
\hline \multirow[b]{2}{*}{ Variables } & \multicolumn{2}{|c|}{ n $(\%)$} & \multirow[b]{2}{*}{$\boldsymbol{P}$} \\
\hline & $\begin{array}{c}\text { Polaprezinc } \\
\text { Plus } \\
\text { Pantoprazole } \\
(\mathrm{N}=104)\end{array}$ & $\begin{array}{l}\text { Rebamipide } \\
\text { Plus } \\
\text { Pantoprazole } \\
(\mathrm{N}=106)\end{array}$ & \\
\hline Age $($ mean \pm SD) $(y)$ & $63.1 \pm 8.8$ & $63.1 \pm 8.8$ & 0.968 \\
\hline Sex & & & 0.747 \\
\hline Male & $78(75.0)$ & $82(77.4)$ & \\
\hline Female & $26(25.0)$ & 24 (22.6) & \\
\hline Histopathology & & & 0.963 \\
\hline Adenoma & $52(50.0)$ & $55(51.9)$ & \\
\hline Carcinoma & $48(46.2)$ & $47(44.3)$ & \\
\hline Other & $4(3.8)$ & $4(3.8)$ & \\
\hline Location & & & 0.252 \\
\hline Upper stomach & $52(50.0)$ & $64(60.4)$ & \\
\hline Middle stomach & $39(37.5)$ & $34(32.1)$ & \\
\hline Lower stomach & $13(12.5)$ & $8(7.5)$ & \\
\hline Gross tumor appearance & & & 0.598 \\
\hline Elevated & $66(63.5)$ & $65(61.3)$ & \\
\hline Flat & $17(16.3)$ & $14(13.2)$ & \\
\hline Depressed & $21(20.2)$ & $27(25.5)$ & \\
\hline $\begin{array}{l}\text { Longest diameter of the } \\
\text { tumor }(\text { mean } \pm \mathrm{SD}) \\
(\mathrm{mm})\end{array}$ & $13.8 \pm 6.5$ & $15.5 \pm 7.1$ & 0.076 \\
\hline $\begin{array}{l}\text { Post-ESD ulcer size } \\
\quad(\text { mean } \pm \text { SD })\left(\mathrm{mm}^{2}\right)\end{array}$ & $865.2 \pm 477.1$ & $888.2 \pm 422.8$ & 0.712 \\
\hline $\begin{array}{l}\text { Specimen size after fixation } \\
\left(\text { mean } \pm \text { SD) }\left(\mathrm{mm}^{2}\right)\right.\end{array}$ & $876.2 \pm 440.6$ & $878.6 \pm 430.2$ & 0.968 \\
\hline En-bloc resection rate & $102(98.1)$ & $105(99.1)$ & 0.620 \\
\hline $\begin{array}{l}\text { Procedure time } \\
\quad(\text { mean } \pm \text { SD) }(\min )\end{array}$ & $29.9 \pm 29.7$ & $26.5 \pm 20.8$ & 0.334 \\
\hline Smoker & $27(26.0)$ & $25(23.6)$ & 0.750 \\
\hline Diabetes mellitus & $9(8.7)$ & $17(16.0)$ & 0.142 \\
\hline Hypertension & $41(39.4)$ & $47(44.3)$ & 0.487 \\
\hline $\begin{array}{l}\text { Helicobacter pylori } \\
\text { infection }\end{array}$ & $35(33.7)$ & $51(48.1)$ & 0.036 \\
\hline $\begin{array}{l}\text { Body mass index } \\
\quad(\text { mean } \pm \mathrm{SD})\left(\mathrm{kg} / \mathrm{m}^{2}\right)\end{array}$ & $24.6 \pm 2.7$ & $24.0 \pm 2.9$ & 0.153 \\
\hline
\end{tabular}

ESD indicates endoscopic submucosal dissection. higher in the PPI plus rebamipide group than in the PPI plus polaprezinc group, both in the full analysis $(91.4 \%$ and $90.3 \%$, respectively; $P=0.523$ ) and per-protocol analysis $(91.1 \%$ and $89.9 \%$, respectively; $P=0.531)$. The percentage of ulcers in the scarring stage at 4 weeks after ESD was not significantly higher in the PPI plus rebamipide group than in the PPI plus polaprezinc group, both in the full analysis $(10.4 \%$ and $9.6 \%$, respectively; $P=0.953)$ and per-protocol analysis $(9.0 \%$ and $7.1 \%$, respectively; $P=0.846$ ) (Table 2 ).

\section{Subgroup Analysis of the Iatrogenic Ulcer Healing Rates}

We performed subgroup analysis to evaluate the risk factors that affect the ulcer healing rate. There were no significant predictive factors for a superior ulcer healing rate between the PPI plus rebamipide group and PPI plus polaprezinc group (Table 3). In addition, we assessed the independent factors for predicting an ulcer healing rate $>90.5 \%$, the mean average healing rate in this study, in multivariate analysis. The procedure time was an independent factor for a higher ulcer healing rate (Table 4).

\section{DISCUSSION}

In this study, the ulcer healing rates at 4 weeks after ESD in the polaprezinc plus PPI treatment group were not inferior compared with those in the rebamipide plus pantoprazole treatment group. There are many reports about the effect of rebamipide on the ulcer healing rate after ESD. ${ }^{3,9,11,12}$ Those studies showed that PPI plus rebamipide is superior to PPI monotherapy for healing artificial ulcers after ESD. However, there are no reports about the effect of polaprezinc on the artificial ulcer healing rate after ESD compared with rebamipide. Polaprezinc, which is a complex chelating agent consisting of L-carnosine and zinc, is widely used as a mucoprotective agent to treat gastric ulcers. ${ }^{13}$ The mechanisms of polaprezinc are independent of prostaglandin, and polaprezinc has antiinflammatory and antioxidant effects. ${ }^{14,15}$ The zinc L-carnosine ameliorates hydrochloric acid-induced mucosal injury by inducing heat shock protein (HSP)-72 expression. ${ }^{16}$ The overexpression of HSP72 protects various organs and cells including gastric mucosal cells against stress conditions. ${ }^{17,18}$ One study compared the effects of polaprezinc with those of rebamipide on gastric mucosal protection. ${ }^{19}$ Polaprezinc showed gastric mucosal protection similar to rebamipide by reducing the levels of inflammatory cytokines and increasing the expression of antioxidant enzymes, growth factors, and HSP. In addition, polaprezinc is helpful for facilitating heal in various conditions, such as radiation, chemotherapy injury, liver fibrosis, and inflammatory bowel disease. ${ }^{20}$ Polaprezinc was used to heal iatrogenic ulcers after ESD in only 1 study. ${ }^{8}$ The PPI plus polaprezinc group showed significantly better ulcer healing and more prevention of protrusion of the ulcer base, indicating that this group had a higher quality of ulcer healing than the PPI monotherapy group.

In this study, the PPI plus polaprezinc group showed noninferiority in ulcer healing compared with the PPI plus rebamipide group. In addition, the only predictor for a higher than average ulcer healing rate was the short procedure time. The long procedure time was an independent risk factor for a low ulcer healing rate, and this result might be associated with the use of more electrocautery during ESD. Electrocautery has been known to result in proper muscle damage. ${ }^{21}$ Therefore, electrocautery caused delayed ulcer healing after ESD. ${ }^{22}$ In addition, the patients in the PPI plus rebamipide group took $100 \mathrm{mg}$ rebamipide 3 times a day 


\begin{tabular}{|c|c|c|c|c|c|c|}
\hline \multirow[b]{2}{*}{ Variables } & \multicolumn{3}{|c|}{ Intent-to-treat Analysis } & \multicolumn{3}{|c|}{ Per-protocol Analysis } \\
\hline & $\begin{array}{c}\begin{array}{c}\text { Polaprezinc+PPI } \\
(\mathrm{n}=104)\end{array} \\
\end{array}$ & $\begin{array}{c}\text { Rebamipide+PPI } \\
(\mathrm{n}=106)\end{array}$ & $\boldsymbol{P}$ & $\begin{array}{c}\text { Polaprezinc+PPI } \\
(n=99)\end{array}$ & $\begin{array}{c}\text { Rebamipide+PPI } \\
(\mathrm{n}=\mathbf{1 0 0})\end{array}$ & $\boldsymbol{P}$ \\
\hline \multicolumn{7}{|c|}{ Longest diameter of the ulcer $(\mathrm{mm})$} \\
\hline Initial & $35.5 \pm 9.4$ & $36.0 \pm 8.8$ & 0.668 & $35.5 \pm 9.6$ & $35.7 \pm 8.7$ & 0.838 \\
\hline 4 wk after ESD & $11.8 \pm 8.1$ & $12.0 \pm 8.2$ & 0.917 & $12.0 \pm 8.1$ & $12.1 \pm 8.3$ & 0.892 \\
\hline \multicolumn{7}{|c|}{ Area of the ulcer $\left(\mathrm{mm}^{2}\right)$} \\
\hline Initial & $865.2 \pm 477.1$ & $888.2 \pm 422.8$ & 0.712 & $866.0 \pm 488.5$ & $871.9 \pm 415.7$ & 0.926 \\
\hline 4 wk after ESD & $96.6 \pm 155.4$ & $93.4 \pm 140.9$ & 0.881 & $99.9 \pm 159.4$ & $97.2 \pm 145.8$ & 0.904 \\
\hline $\begin{array}{l}\text { Healing rate at } \\
4 \mathrm{wk}(\%)\end{array}$ & 90.3 & 91.4 & 0.523 & 89.9 & 91.1 & 0.531 \\
\hline Scar stages at $4 \mathrm{wk}$ & $10(9.6)$ & $11(10.4)$ & 0.953 & $7(7.1)$ & $9(9.0)$ & 0.846 \\
\hline
\end{tabular}

ESD indicates endoscopic submucosal dissection; PPI, proton pump inhibitor.

and the patients in the PPI plus polaprezinc group took $75 \mathrm{mg}$ of polaprezinc twice a day. Therefore, one advantage of polaprezinc compared with rebamipide is its twice a day dosing. However, the cost of polaprezinc was 216 Korean won (KRW) and rebamipide $99 \mathrm{KRW}$, respectively. Therefore, the daily cost of polaprezinc was $432 \mathrm{KRW}$ and rebamipide $297 \mathrm{KRW}$. The cost of polaprezinc was higher than that of rebamipide.

There is no fundamental guideline to determine the appropriate PPI dose and treatment duration for iatrogenic ulcer healing. Some studies reported variable treatment duration (1, 2, 4, and $8 \mathrm{wk}$ ) and PPI dose (half or full dose) for ESD ulcers. ${ }^{23-26}$ The predictors for delayed ulcer healing have been known to be the tumor location, comorbidity, larger tumor size, and electrocoagulation. ${ }^{22,27}$ In this study, the patients took oral PPI plus rebamipide or polaprezinc for 4 weeks. The average ulcer healing rate was $90.5 \%$. This result was comparable with that of previous studies. ${ }^{9,19}$ However, the proportion of patients with scarring stage was low $(16 / 199,8.0 \%)$. Therefore, we could not evaluate the quality of ulcer healing well. Shin et $\mathrm{al}^{9}$ reported that the scarring stage rate at 4 weeks was 20.8\% (53/255) after ESD in both the PPI and rebamipide and PPI monotherapy groups. Furthermore, Nakamura et $\mathrm{al}^{12}$ showed that the scarring stage rate at 4 weeks was $14.4 \%$ (15/ 104) after ESD in both the PPI and rebamipide and PPI monotherapy groups. Although the scarring stage rate at 4

TABLE 3. Predictors of Higher Ulcer Healing Rate at 4 Weeks After ESD in Univariate Analysis

\begin{tabular}{|c|c|c|c|c|c|c|}
\hline \multirow[b]{2}{*}{ Variables } & \multicolumn{3}{|c|}{ Intent-to-treat Analysis [n (\%)] } & \multicolumn{3}{|c|}{ Per-protocol Analysis [n (\%)] } \\
\hline & $\begin{array}{c}\text { Polaprezinc+PPI } \\
(n=104)\end{array}$ & $\begin{array}{c}\text { Rebamipide+PPI } \\
(n=106)\end{array}$ & $\boldsymbol{P}$ & $\begin{array}{c}\text { Polaprezinc+PPI } \\
(\mathrm{n}=99)\end{array}$ & $\begin{array}{c}\text { Rebamipide+PPI } \\
(\mathrm{n}=100)\end{array}$ & $\boldsymbol{P}$ \\
\hline \multicolumn{7}{|c|}{ Initial longest diameter of the ulcer (mm) } \\
\hline$<35$ & $49(91.4)$ & 48 (92.6) & 0.575 & $47(91.1)$ & $46(92.4)$ & 0.579 \\
\hline$\geq 35$ & $55(88.2)$ & $58(90.0)$ & 0.501 & $52(87.7)$ & $54(89.5)$ & 0.512 \\
\hline \multicolumn{7}{|c|}{ Initial area of the ulcer $\left(\mathrm{mm}^{3}\right)$} \\
\hline$<800$ & $51(90.2)$ & $54(92.4)$ & 0.316 & $49(90.0)$ & $52(92.2)$ & 0.313 \\
\hline$\geq 800$ & $53(89.2)$ & $52(89.9)$ & 0.803 & $50(88.7)$ & $48(89.3)$ & 0.830 \\
\hline \multicolumn{7}{|c|}{ Procedure time (min) } \\
\hline$<21$ & $46(93.5)$ & $57(92.1)$ & 0.540 & $43(93.2)$ & $54(91.8)$ & 0.566 \\
\hline$\geq 21$ & $58(86.7)$ & $49(90.1)$ & 0.196 & $56(86.4)$ & 46 (89.6) & 0.239 \\
\hline \multicolumn{7}{|l|}{$\operatorname{Age}(y)$} \\
\hline$<64$ & $47(90.3)$ & $56(90.4)$ & 0.962 & $46(90.1)$ & $53(90.0)$ & 0.948 \\
\hline$\geq 64$ & $57(89.2)$ & $50(92.1)$ & 0.286 & $53(88.6)$ & $47(91.8)$ & 0.257 \\
\hline \multicolumn{7}{|l|}{ Pathology } \\
\hline Adenoma & $52(91.0)$ & $55(92.1)$ & 0.609 & $48(90.4)$ & $53(91.9)$ & 0.515 \\
\hline Carcinoma & $48(89.3)$ & 47 (89.6) & 0.900 & $47(89.1)$ & $43(89.0)$ & 0.955 \\
\hline Other & $4(78.3)$ & $4(96.9)$ & 0.192 & $4(78.3)$ & $4(96.9)$ & 0.192 \\
\hline \multicolumn{7}{|l|}{ Location } \\
\hline Antrum & $52(89.5)$ & $64(92.3)$ & 0.235 & $49(89.0)$ & $59(91.8)$ & 0.243 \\
\hline Other & $52(89.9)$ & $42(89.5)$ & 0.880 & $50(89.6)$ & $41(89.4)$ & 0.939 \\
\hline \multicolumn{7}{|c|}{ Helicobacter pylori infection } \\
\hline No & $69(89.1)$ & $55(90.6)$ & 0.546 & $64(88.4)$ & $52(90.2)$ & 0.490 \\
\hline Yes & $35(91.0)$ & $51(91.8)$ & 0.720 & $35(91.0)$ & $48(91.5)$ & 0.840 \\
\hline \multicolumn{7}{|c|}{ Combined diseases } \\
\hline No & $56(88.2)$ & $50(90.6)$ & 0.377 & $54(87.8)$ & $48(90.3)$ & 0.376 \\
\hline Yes & $48(91.5)$ & $56(91.7)$ & 0.920 & $45(91.1)$ & $52(91.3)$ & 0.927 \\
\hline
\end{tabular}

ESD indicates endoscopic submucosal dissection; PPI, proton pump inhibitor. 
TABLE 4. Independent Predictors for Higher Than Average Ulcer Healing Rates ( $\geq 90.5 \%$ ) at 4 Weeks After ESD in Logistic Regression Analysis

\begin{tabular}{|c|c|c|c|c|c|}
\hline \multirow[b]{2}{*}{ Variables } & \multicolumn{3}{|c|}{ Univariate Analysis } & \multicolumn{2}{|c|}{ Multivariate Analysis } \\
\hline & $\begin{array}{c}\text { Ulcer Healing Rate } \\
<90.5 \%\end{array}$ & $\begin{array}{c}\text { Ulcer Healing Rate } \\
\mathbf{2 9 0 . 5 \%} \\
\end{array}$ & $\boldsymbol{P}$ & $\begin{array}{l}\text { Odds Ratio } \\
(95 \% \text { CI })\end{array}$ & $\boldsymbol{P}$ \\
\hline Age (y) & $61.5 \pm 7.6$ & $63.7 \pm 9.1$ & 0.097 & $1.039(0.999-1.080)$ & 0.056 \\
\hline Underlying diseases & $28 / 59(47.5)$ & $76 / 151(50.3)$ & 0.760 & $0.704(0.353-1.404)$ & 0.318 \\
\hline Ulcer location (antrum) & $29 / 59(49.2)$ & $83 / 151(55.0)$ & 0.538 & $0.824(0.410-1.655)$ & 0.586 \\
\hline Initial longest diameter of the ulcer & $37.9 \pm 10.2$ & $34.9 \pm 8.5$ & 0.054 & $0.972(0.874-1.080)$ & 0.593 \\
\hline Initial area of the ulcer & $962.4 \pm 463.7$ & $843.4 \pm 441.0$ & 0.085 & $1.000(0.998-1.003)$ & 0.649 \\
\hline Carcinoma & $29 / 59(49.2)$ & $66 / 151(43.7)$ & 0.538 & $0.801(0.413-1.553)$ & 0.511 \\
\hline Helicobacter pylori infection & $25 / 59(42.4)$ & $61 / 151(40.4)$ & 0.876 & $1.079(0.549-2.120)$ & 0.825 \\
\hline Procedure time & $38.8 \pm 36.5$ & $24.1 \pm 18.3$ & 0.004 & $0.975(0.958-0.993)$ & 0.006 \\
\hline $\mathrm{PPI}+$ polaprezinc & 25/59 (42.4) & $81 / 151(53.6)$ & 0.168 & $0.648(0.340-1.233)$ & 0.186 \\
\hline
\end{tabular}

CI indicates confidence interval; ESD, endoscopic submucosal dissection; PPI, proton pump inhibitor.

weeks herein was lower than that in other studies, the incidence of bleeding was low $(1 / 210,0.4 \%)$. This low bleeding rate might be due to complete electrocoagulation of exposed vessels of the iatrogenic ulcer.

In a previous study, polaprezinc showed better ulcer healing and prevention of protrusion of the ulcer base after ESD. ${ }^{8}$ In this study, we did not evaluate the effects of quality of ulcer healing after ESD in the polaprezinc plus PPI group because the proportion of patients with ulcer scarring stage was low. Further studies will be needed to investigate the effects of polaprezinc on the quality of ulcer healing.

In conclusion, polaprezinc plus PPI treatment showed noninferiority to rebamipide plus PPI treatment in the 4-week ESD-induced ulcer healing rate.

\section{REFERENCES}

1. Gotoda T, Ho KY, Soetikno R, et al. Gastric ESD: current status and future directions of devices and training. Gastrointest Endosc Clin N Am. 2014;24:213-233.

2. Okada K, Yamamoto Y, Kasuga A, et al. Risk factors for delayed bleeding after endoscopic submucosal dissection for gastric neoplasm. Surg Endosc. 2011;25:98-107.

3. Wang J, Guo X, Ye C, et al. Efficacy and safety of proton pump inhibitors (PPIs) plus rebamipide for endoscopic submucosal dissection-induced ulcers: a meta-analysis. Intern Med. 2014:53:1243-1248.

4. Naito Y, Yoshikawa T. Rebamipide: a gastrointestinal protective drug with pleiotropic activities. Expert Rev Gastroenterol Hepatol. 2010;4:261-270.

5. Kleine A, Kluge S, Peskar BM. Stimulation of prostaglandin biosynthesis mediates gastroprotective effect of rebamipide in rats. Dig Dis Sci. 1993;38:1441-1449.

6. Naito Y, Yoshikawa T, Tanigawa T, et al. Hydroxyl radical scavenging by rebamipide and related compounds: electron paramagnetic resonance study. Free Radic Biol Med. 1995;18:117-123.

7. Murakami-Nakayama M, Tsubota M, Hiruma S, et al. Polaprezinc attenuates cyclophosphamide-induced cystitis and related bladder pain in mice. J Pharmacol Sci. 2015;127:223-228.

8. Inaba $\mathrm{T}$, Ishikawa $\mathrm{S}$, Toyokawa $\mathrm{T}$, et al. Basal protrusion of ulcers induced by endoscopic submucosal dissection (ESD) during treatment with proton pump inhibitors, and the suppressive effects of polaprezinc. Hepatogastroenterology. 2010; 57:678-684.

9. Shin WG, Kim SJ, Choi MH, et al. Can rebamipide and proton pump inhibitor combination therapy promote the healing of endoscopic submucosal dissection-induced ulcers? A randomized, prospective, multicenter study. Gastrointest Endosc. 2012;75: 739-747.

10. Sakita T, Fukutomi H, Yoshitoshi Y. Ulcer of the stomach and duodenum; 1971.

11. Araki H, Kato T, Onogi F, et al. Combination of proton pump inhibitor and rebamipide, a free radical scavenger, promotes artificial ulcer healing after endoscopic submucosal dissection with dissection size $>40 \mathrm{~mm}$. J Clin Biochem Nutr. 2012;51: $185-188$.

12. Nakamura K, Ihara E, Akiho $\mathrm{H}$, et al. Limited effect of rebamipide in addition to proton pump inhibitor (PPI) in the treatment of post-endoscopic submucosal dissection gastric ulcers: a randomized controlled trial comparing PPI plus rebamipide combination therapy with PPI monotherapy. Gut Liver. 2016;10:917-924.

13. Liu ZY, Xie WB, Ru Li M, et al. Effects of polaprezinc on gastric mucosal damage and neurotransmitters in a rat model of chemotherapy-induced vomiting. J Int Med Res. 2018;46: 2436-2444.

14. Opoka W, Adamek D, Plonka M, et al. Importance of luminal and mucosal zinc in the mechanism of experimental gastric ulcer healing. J Physiol Pharmacol. 2010;61:581-591.

15. Wada I, Otaka M, Jin M, et al. Expression of HSP72 in the gastric mucosa is regulated by gastric acid in rats-correlation of HSP72 expression with mucosal protection. Biochem Biophys Res Commun. 2006;349:611-618.

16. Mikami K, Otaka M, Watanabe D, et al. Zinc L-carnosine protects against mucosal injury in portal hypertensive gastropathy through induction of heat shock protein 72. $J$ Gastroenterol Hepatol. 2006;21:1669-1674.

17. Kiang JG, Wang XD, Ding XZ, et al. Heat shock inhibits the hypoxia-induced effects on iodide uptake and signal transduction and enhances cell survival in rat thyroid FRTL-5 cells. Thyroid. 1996;6:475-483.

18. Mestril R, Chi SH, Sayen MR, et al. Isolation of a novel inducible rat heat-shock protein (Hsp70) gene and its expression during ischemia hypoxia and heat-shock. Biochem J. 1994;298: 561-569.

19. Choi HS, Lim JY, Chun HJ, et al. The effect of polaprezinc on gastric mucosal protection in rats with ethanol-induced gastric mucosal damage: comparison study with rebamipide. Life Sci. 2013;93:69-77.

20. Ooi TC, Chan KM, Sharif R. Antioxidant, anti-inflammatory, and genomic stability enhancement effects of zinc l-carnosine: a potential cancer chemopreventive agent? Nutr Cancer. 2017;69: 201-210.

21. Horikawa Y, Mimori N, Mizutamari H, et al. Proper muscle layer damage affects ulcer healing after gastric endoscopic submucosal dissection. Dig Endosc. 2015;27:747-753. 
22. Lim JH, Kim SG, Choi J, et al. Risk factors of delayed ulcer healing after gastric endoscopic submucosal dissection. Surg Endosc. 2015;29:3666-3673.

23. Higuchi $\mathrm{K}$, Takeuchi T, Uedo N, et al. Efficacy and safety of 1-week Helicobacter pylori eradication therapy and 7-week rebamipide treatment after endoscopic submucosal dissection of early gastric cancer in comparison with 8-week PPI standard treatment: a randomized, controlled, prospective, multicenter study. Gastric Cancer. 2015;18:612-617.

24. Park JH, Baek EK, Choi CH, et al. Comparison of the efficacy of 4- and 8-week lansoprazole treatment for ESD-induced gastric ulcers: a randomized, prospective, controlled study. Surg Endosc. 2014;28:235-241.
25. Kawano S, Okada H, Kawahara Y, et al. Proton pump inhibitor dose-related healing rate of artificial ulcers after endoscopic submucosal dissection: a prospective randomized controlled trial. Digestion. 2011;84:46-53.

26. Arai M, Matsumura T, Okimoto K, et al. Two-week treatment with proton pump inhibitor is sufficient for healing post endoscopic submucosal dissection ulcers. World $J$ Gastroenterol. 2014;20:16318-16322.

27. Yoshizawa Y, Sugimoto M, Sato Y, et al. Factors associated with healing of artificial ulcer after endoscopic submucosal dissection with reference to Helicobacter pylori infection, CYP2C19 genotype, and tumor location: multicenter randomized trial. Dig Endosc. 2016;28:162-172. 